\title{
Identificación de macro invertebrados bentónicos en los ríos: Pindo Mirador, Alpayacu y Pindo Grande; determinación de su calidad de agua Alexandra Endara ${ }^{1}$
}

\begin{abstract}
Resumen
En un estudio realizado en el mes de mayo del 2012, utilizando una red Surber de $30 \times 30 \mathrm{~cm}$ de área de superficie y 0,5 mm de abertura de malla, en los ríos: Pindo Mirador, Pindo Grande y Alpayacu, ubicados en el sector de Mera, provincia de Pastaza de la Amazonía ecuatoriana, se analizó la diversidad y la abundancia de los macro invertebrados bentónicos utilizando los índices BMWP/Col y EPT. Se logró determinar que la calidad del agua de los ríos Pindo Mirador y Pindo Grande es buena mientras la del río Alpayacu es mala. Este estudio da una idea general de la situación ambiental de los ecosistemas lóticos cercanos a la Estación Biológica Pindo Mirador. Los resultados de ésta investigación permiten determinar la importancia de las micro-cuencas de los ríos Pindo Mirador y Pindo Grande como fuente de agua para las poblaciones que se encuentran río abajo, así como el modo en el cual la presencia o ausencia de organismos bioindicadores (macroinvertebrados) indica la calidad del agua y de los bosques de la micro-cuenca.
\end{abstract}

Palabras clave:

Macro-invertebrados, Índices BMWP/Col y EPT, Microcuenca y Calidad del agua.

\begin{abstract}
In May 2012, using a $30 \times 30 \mathrm{~cm}$ surface and $0.5 \mathrm{~mm}$ aperture Surber net in the rivers: Pindo Mirador, Pindo Grande and Alpayacu, located in Mera, Pastaza, Ecuadorian Amazonia, the diversity and abundance of macro invertebrates was analyzed applying the BMWP/Col and the EPT indexes. It was determined the good Pindo Mirador, Pindo Grande and Alpayacu rivers' water quality, as well as the bad Alpayacu river's water quality. This work provides an overview of the environmental situation of aquatic ecosystems near Pindo Mirador Biological Station, it highlights the importance of the rivers' micro-watersheds near Pindo Mirador Biological Station, used as water source by the populations living downstream, and shows how macro invertebrates can be used as bioindicators for the water and forests quality.
\end{abstract}

\section{Keywords:}

Macro-invertebrates, BMWP/Col and EPT index, Water Quality.

\section{Introducción}

Los macroinvertebrados acuáticos se definen como aquellos organismos que al menos durante algún estadio de su ciclo de vida, vivan exclusivamente en el ambiente acuático y que se puedan ver a simple vista, es decir, que tengan un tamaño superior a $0.5 \mathrm{~mm}$ de longitud (Roldán, 1988). Estos organismos (70 - 90\% insectos) son usados con éxito como bioindicadores porque se desenvuelven durante una gran parte de su vida en los medios acuáticos. Generalmente son abundantes, relativamente sedentarios, son consumidores primarios y secundarios en el proceso de la materia orgánica, su colecta es simple y barata, son fáciles de ver y ofrecen información de largos períodos de tiempo.

\footnotetext{
${ }^{1}$ Universidad Tecnológica Equinoccial, Facultad de Ciencias de la Ingeniería, Quito - Ecuador (aendara@ute.edu.ec).
} 
La presencia de una comunidad de macro invertebrados en un cuerpo de agua determinado, es un índice inequívoco de las condiciones que allí están dominando y de que las fluctuaciones de contaminación que puedan presentarse, no son lo suficientemente fuertes como para provocar un cambio significativo en la misma. Además de eso, se deben considerar otros factores importantes relacionados con la distribución de la composición taxonómica, como son las características propias de profundidad, ancho, turbidez, luz, detritos, turbulencia e inconstancia del substrato del cuerpo de agua y vegetación de las orillas (Roldán, 2003).

En las últimas décadas los ecosistemas acuáticos han tenido una fuerte presión humana, debido a las actividades agrícolas, deforestación, fragmentación del hábitat, cambios del sustrato por la remoción y extracción de materiales, ingreso de aguas servidas, actividad petrolera, etc., todo esto afectando la calidad del agua (Dominguez y Fernández, 2009).

Los índices más ampliamente usados para sistemas lóticos (ríos y riachuelos) y lénticos (lagos, lagunas) son el Índice BMWP/Col (BiologicalMonitoringWaterParty/Colombia) (Roldán, 2003) y el Índice EPT (Ephemeróptera, Plecóptera y Trichoptera) (Carrera y Fierro, 2001), los cuales son útiles en el análisis de la calidad del agua, debido a que necesitan bajo nivel taxonómico (Familia), bajo costo en términos de tiempo (identificación de insectos) y dinero, convirtiéndose en metodologías rápidas y útiles para ser utilizadas en la fiscalización por parte de algún organismo público que requiera en poco tiempo y de una forma acertada evaluar la calidad del agua de una cuenca hidrográfica determinada (Roldán, 2003).

Tomando en consideración lo antes expuesto se procedió a examinar los macro invertebrados bentónicos de los ríos cercanos a la Estación Biológica Pindo Mirador (Pindo Grande, Pindo Mirador y Alpayacu) para analizar su calidad de agua.

\section{Metodología}

\section{Fase de campo}

En la fase de campo, a las 10:00 am, se realizó un muestreo de 10 puntos elegidos al azar a lo largo de 50 metros a las orillas de los ríos Pindo Mirador, Pindo Grande y Alpayacu.

El muestreo se realizó utilizando una red Surber de 30 x $30 \mathrm{~cm}$ de área de superficie y 0,5 mm de abertura de malla. Las muestras colectadas fueron colocadas en bandejas de loza, donde fueron separados los macro invertebrados del sedimento y de otras partículas. Éstos fueron fijados en alcohol al $70 \%$ y posteriormente se llevaron a la Estación Biológica Pindo Mirador para su identificación. 


\section{Fase de Laboratorio}

En el laboratorio, las muestras fueron separadas y los macro invertebrados fueron separados del sustrato con la ayuda de una pinza tipo relojero y posteriormente fueron colocados en un estero microscopio para su identificación; se procuró tomar a los insectos de su abdomen, debido a que así, estos sufren menos daño en su cuerpo y especialmente en alas y cabeza, por tanto, su posterior identificación en el laboratorio es menos dificultosa.

Los macro invertebrados colectados se guardaron en tubos de ensayo vacutainer con alcohol al $70 \%$. La identificación de los ejemplares se la realizó a través de claves dicotómicas, usadas para la entomofauna acuática neotropical (Domínguez y Fernández, 2009; Fernández. y Domínguez, 2001; Manzo, 2005; Merritt y Cummins, 1988; Roldán, 1988; Salles, 2006).

Se evaluaron los siguientes parámetros de las comunidades de macro invertebrados acuáticos estudiados:

- Riqueza (S).- Número total de morfoespecies en cada punto de muestreo.

- Abundancia (N).- Número total de individuos registrados en cada punto de muestreo.

- Abundancia relativa (\%).- Número de individuos de cada especie multiplicado por cien y dividido por la abundancia total registrada en cada cuerpo de agua. Además se utilizó la siguiente escala: Raro (1 a 3 individuos), Común (4 - 9 individuos), Abundante (10 - 49 individuos) y Dominantes (50 o más individuos) (EPA, 1989)

- Morfoespecies indicadoras.- Se uso la clasificación de Roldán (2003), que considera a las morfoespecies con puntajes BMWP/Col de 8 -10 como de Clase I = Indicadores de Buena calidad; las morfoespecies con puntajes BMWP/Col de 4-7 como de Clase II = Indicadores de Mediana Calidad y las morfoespecies con puntajes BMWP/Col de $1-3$ como de Clase III = Indicadores de Mala Calidad.

- Para determinar la calidad del agua se utilizó el Índice BMWP/Col (Biological Monitoring Working Party para Colombia), el cual da valores de 1 a 10 a los macro-invertebrados identificados a nivel de familia. Las familias que no toleran la pérdida de la calidad de agua tienen puntajes altos, mientras que familias que toleran la pérdida de calidad tienen puntajes bajos. La suma total de los puntajes de todas las familias encontradas en un sitio proporcionan el valor de la calidad del agua (Roldán, 2003).

\section{3. Área de estudio}

Las muestras se colectaron el 5 de mayo del 2012, en las cuencas de los ríos: Pindo - Mirador, Pindo Grande y Alpayacu ubicados en el Cantón Mera de la Provincia de Pastaza. La temperatura promedio en la zona es de 20 y $25^{\circ} \mathrm{C}$, su clima es mesotérmico, perhúmedo y de permanente lluvia, las precipitaciones anuales tienen un promedio de $4500 \mathrm{~mm} 3$ siendo la humedad constante en el tiempo. 
El río Pindo Mirador en el sector de la colecta tuvo una anchura promedio de 10,5 m. y una profundidad aproximada de $45 \mathrm{~cm}$. El sustrato del río es rocoso pedregoso con presencia de necróforos (materia vegetativa en descomposición), la formación vegetal que presenta es bosque siempre verde pie montano, en sus riveras se observa vegetación típica de este tipo de bosque, dando sombra. Las coordenadas en el sitio del muestreo fueron de $1^{\circ} 27^{\prime} 47.2^{\prime \prime} S 78^{\circ} 5^{\prime} 14.3^{\prime \prime} \mathrm{O}$ a los 1153 msnm.

En el río Pindo - Grande se tomaron las muestras en las siguientes coordenadas geográficas: Latitud, 17M08253, Longitud: UTM 9838684 a una altura de $1040 \mathrm{msnm}$. El río en el sitio del muestreo tuvo $8,8 \mathrm{~m}$. de ancho y $40 \mathrm{~cm}$. de profundidad, con agua turbia, no presentó olor, tuvo un sustrato pedregoso arenoso con gran cantidad de piedras. La vegetación a la orilla es secundaria (Cecropias), bosque que fue talado y luego se regeneró. Alrededor del río también se pudieron observar pastizales, la vegetación no es variada por la presencia de viveros.

El río Alpayacu es un afluente del río Pastaza que se ubica en Mera en la provincia de Pastaza a 18 km. del Puyo, en la vía a Baños. El Río Alpayacu en el sitio del muestreo es un río de segundo orden con una superficie de 11 hectáreas con vegetación natural, plantas pioneras y epifitas. Se pudo observar la presencia de Guarumos (Cecropias) y Caña Brava (Bombacáceas), los muestreos se realizaron en las siguientes coordenadas: Longitud 17m0822384, Latitud UTM 9837088, a una altitud $1053 \mathrm{msnm}$. El momento del muestreo el tiempo fue nubloso con una cobertura de 4/4 y la presencia de lluvia ligera. El río fue de fácil acceso con un ancho aproximado de 4-5 m. y una profundidad variable según la localización del río. En el sitio del muestreo el río tenía gran cantidad de materia orgánica debido a desagües y aguas servidas que desembocan en el río, presencia de volquetas para extracción pétrea, suelo pedregoso y arenoso. Además de la presencia de gallinazos debido a la presencia de carroña.

\section{Resultados y discusión}

La composición taxonómica del río Pindo - Mirador fue de 15 Familias, repartidas en 9 órdenes, de los cuales 7 corresponden a la clase Insecta según se muestra en la Tabla 1.

El análisis de EPT se realizó mediante la utilización de macroinvertebrados considerados indicadores de la calidad de agua, debido a que son más sensibles a la contaminación. En primer lugar se coloca en una columna la clasificación de organismos, en una segunda columna la abundancia y una última columna con los EPT presentes como se muestra en la Tabla 2. Posteriormente los EPT presentes se dividen por la abundancia total, obteniendo un valor, el cual se lleva a una tabla de calificaciones de calidad de agua que va de muy buena a mala calidad según la Tabla 3 y la Tabla 4. 
Tabla 1. Riqueza y Abundancia de Macroinvertebrados del Punto de muestreo del Río Pindo - Mirador (Pastaza - Mera).

\begin{tabular}{|c|c|c|c|}
\hline & & RIQUEZA & ABUNDANCIA \\
\hline CLASE & ORDEN & FAMILIA & $\begin{array}{l}\text { NÚMERO DE } \\
\text { INDIVIDUOS }\end{array}$ \\
\hline Turbelaria & Tricladia & Planarlidae & 5 \\
\hline \multirow[t]{13}{*}{ Insecta } & \multirow[t]{2}{*}{ Ephemeroptera } & Baetidae & 18 \\
\hline & & Leptophlebiidae & 2 \\
\hline & \multirow[t]{2}{*}{ Odonata } & Polythoridae & 2 \\
\hline & & Libellulidae & 3 \\
\hline & Lepidoptera & Pyralidae & 2 \\
\hline & Hemiptera & Naucoridae & 3 \\
\hline & \multirow[t]{4}{*}{ Coleoptera } & Psephenidae & 2 \\
\hline & & Ptilodactylidae & 1 \\
\hline & & Elmidae & 2 \\
\hline & & Hidrophilidae & 1 \\
\hline & Trichoptera & Hydropsychidae & 8 \\
\hline & \multirow[t]{2}{*}{ Diptera } & Tipulidae & 3 \\
\hline & & Chironomidae & 4 \\
\hline Crustacea & Decapodo & Palaemonidae & 1 \\
\hline TOTAL & & & 57 \\
\hline
\end{tabular}

Tabla 2. Índices biológicos: EPT (Ephemeroptera, Plecoptera, Trichoptera)

\begin{tabular}{|c|c|c|c|c|c|}
\hline CLASE & ORDEN & FAMILIA & $\begin{array}{l}\text { CALIFICACIÓN } \\
\text { INDICE } \\
\text { BMWP/Col }\end{array}$ & INDICE EPT & \\
\hline Turbelaria & Tricladia & Planarlidae & 7 & & \\
\hline \multirow[t]{13}{*}{ Insecta } & \multirow[t]{2}{*}{ Ephemeroptera } & Baetidae & 7 & 18 & \\
\hline & & Leptophlebiidae & 9 & 2 & \\
\hline & \multirow[t]{2}{*}{ Odonata } & Polythoridae & 10 & & \\
\hline & & Libellulidae & 6 & & \\
\hline & Lepidoptera & Pyralidae & 5 & & \\
\hline & Hemiptera & Naucoridae & 7 & & \\
\hline & \multirow[t]{4}{*}{ Coleoptera } & Psephenidae & 10 & & \\
\hline & & Ptilodactylidae & 10 & & \\
\hline & & Elmidae & 6 & & \\
\hline & & Hidrophilidae & 7 & & \\
\hline & Trichoptera & Hydropsychidae & 7 & 8 & \\
\hline & \multirow[t]{2}{*}{ Diptera } & Tipulidae & 3 & & \\
\hline & & Chironomidae & 2 & & \\
\hline Crustacea & Decapodo & Palaemonidae & 8 & & \\
\hline TOTAL & & & 104 & 28 & $\begin{array}{c}\text { AGUA } \\
\text { REGULAR }\end{array}$ \\
\hline
\end{tabular}

Tabla. 3. Índice EPT Y BMWP/COL.

\begin{tabular}{|c|c|c|c|c|}
\hline CLASE & CALIDAD & BMWP/Col & SIGNIFICADO & COLOR \\
\hline I & Buena & $\geq 150,101-120$ & $\begin{array}{c}\text { Aguas muy limpias } \\
\text { a limpias }\end{array}$ & \\
\hline II & Aceptable & $61-100$ & $\begin{array}{c}\text { Aguas ligeramente } \\
\text { contaminadas }\end{array}$ & \\
\hline III & Dudosa & $36-60$ & $\begin{array}{c}\text { Aguas } \\
\text { moderadamente } \\
\text { contaminadas }\end{array}$ & \\
\hline IV & Crítica & $16-35$ & $\begin{array}{c}\text { Aguas muy } \\
\text { contaminadas }\end{array}$ & \\
\hline V & Muy crítica & $\leq 15$ & $\begin{array}{c}\text { Aguas fuertemente } \\
\text { contaminadas }\end{array}$ & \\
\hline
\end{tabular}


Tabla 4. Cálculo de los Índices EPT yBMWP/COL.

\begin{tabular}{|c|c|c|}
\hline \multicolumn{2}{|c|}{ CALIDAD DE AGUA } & \\
\hline $75 \%-100 \%$ & MUY BUENA & \\
\hline $50 \%-74 \%$ & BUENA & $28 / 57=0,49$ \\
\hline $25 \%-49 \%$ & REGULAR & $0,49 * 100=49 \%$ \\
\hline $0 \%-24 \%$ & MALA & \\
\hline
\end{tabular}

De lo observado en las tablas 1, 2, 3 y 4 se puede determinar que en el río Pindo Mirador existe una gran biodiversidad y una buena calidad de agua; por lo tanto se debe controlar el impacto ambiental en éste para prevenir la reducción del ecosistema y contaminación del agua. Por ejemplo si se va a trabajar cerca del río, no botar materia orgánica, utilizar para la reforestación plantas endémicas, no introducir especies alóctonas de flora y fauna y establecer medidas de restricción por medio de ordenanzas públicas a constructoras y empresas, para evitar la ubicación de las mismas cerca del cuerpo de agua, ya que destruyen el ecosistema. De esta manera se garantizaría que la micro cuenca del río Pindo Mirador se mantenga en buenas condiciones como hasta el momento arrojan los resultados del análisis realizado.

La composición taxonómica del río Pindo Grande fue de 18 Familias, repartidas en 9 órdenes, de los cuales 6 corresponden a la clase Insecta, según la Tabla 5.

Tabla 5. Riqueza y Abundancia de Macroinvertebrados del Punto de muestreo del Río Pindo Grande

\begin{tabular}{|c|c|c|c|c|c|}
\hline CLASE & ORDEN & FAMILIA & ABUNDANCIA & $\begin{array}{c}\text { BMWP/ } \\
\text { COL }\end{array}$ & EPT \\
\hline Tubellaria & Tricladida & Planariidae & 1 & 7 & \\
\hline Oligochaeta & Haplotaxida & Tubificidae & 2 & 1 & \\
\hline Insecta & Ephemeroptera & Baetidae & 11 & 7 & 7 \\
\hline & & Leptohyphidae & 18 & 7 & 7 \\
\hline & & Leptophlebiidae & 24 & 9 & 9 \\
\hline & Plecopetera & Perlidae & 2 & 10 & 10 \\
\hline & Hemiptera & Naucoridae & 1 & 7 & \\
\hline & & Veliidae & 1 & 8 & \\
\hline & Coleoptera & Elmidae & 11 & 6 & \\
\hline & & Ptilodactylidae & 8 & 10 & \\
\hline & Trichoptera & Glossosomatidae & 3 & 7 & 7 \\
\hline & & Hidropsychidae & 9 & 7 & 7 \\
\hline & & Elicopsychidae & 6 & 8 & 8 \\
\hline & & Leptoceridae & 9 & 8 & 8 \\
\hline & Diptera & Tipulidae & 1 & 3 & \\
\hline & & Chironomidaae & 1 & 2 & \\
\hline Crustacea & Decapoda & Simuliidae & 4 & 8 & \\
\hline TOTAL & & & 3 & 8 & \\
\hline
\end{tabular}

Mediante los índices BMWP/Col se determino que las aguas del río Pindo Grande son aguas limpias. Con el método EPT se observó que las aguas son de buena calidad, lo cual quiere decir 
que son aptas como fuente para el consumo humano. Las especies abundantes en este río fueron las del orden Ephemeróptera y Trichóptera como se muestra en la Figura 1 y la Figura 2.

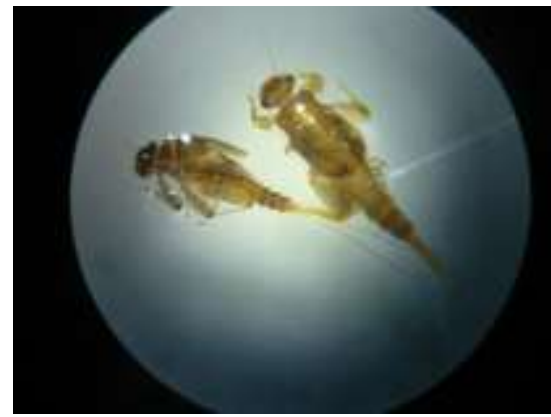

Figura 1. Ephemeróptero

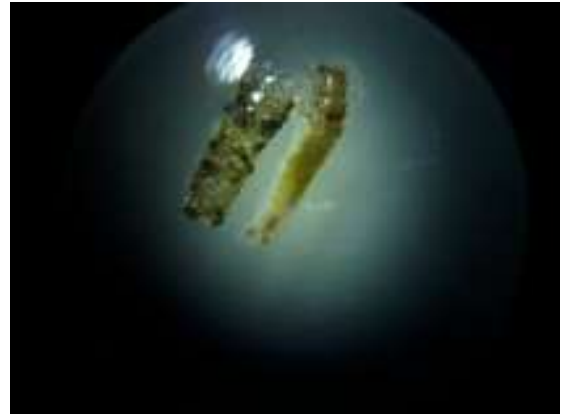

Figura 2. Trichóptero

En las muestras analizadas se obtuvieron: 4 clases, 10 órdenes, 16 familias, 53 individuos según la Tabla 6.

Tabla 6. Riqueza y Abundancia de Macroinvertebrados del Punto de muestreo del Río Alpayacu

\begin{tabular}{|c|c|c|c|c|c|}
\hline Clase & Orden & Familia & $\begin{array}{c}\text { \# de } \\
\text { individuos }\end{array}$ & $\begin{array}{c}\text { Calificación índice } \\
\text { BMWP }\end{array}$ & $\begin{array}{c}\text { Índice } \\
\text { EPT }\end{array}$ \\
\hline Arácnida & Acari & Lymnessiidae & 3 & 10 & \\
\hline \multirow[t]{9}{*}{ Insecta } & Coleoptera & Elmidae & 1 & 6 & \\
\hline & & Ptilodactylidae & 4 & 10 & \\
\hline & Ephemeroptera & Baetidae & 10 & 7 & 10 \\
\hline & & Leptophlebiidae & 3 & 9 & 3 \\
\hline & & Leptohyphidae & 1 & 7 & 1 \\
\hline & Hemiptera & Naucoridae & 12 & 7 & \\
\hline & & Veliidae & 4 & 8 & \\
\hline & Neuroptera & Corydalidae & 1 & 6 & \\
\hline & Odonata & Aeshnidae & 1 & 6 & \\
\hline \multirow[t]{5}{*}{ Clase } & Orden & Familia & \# de individuos & Calificación índice BMWP & $\begin{array}{c}\text { Índice } \\
\text { EPT }\end{array}$ \\
\hline & & Libelluidae & 2 & 6 & \\
\hline & Diptera & Chironomidae & 7 & 2 & \\
\hline & Tricoptera & Hidropsychidae & 1 & 7 & 1 \\
\hline & & Leptoceridae & 1 & 8 & 1 \\
\hline Oligochaeta & Haplotaxida & Tubificidae & 1 & 1 & \\
\hline Turbelaria & Tricladida & Planariidae & 1 & 7 & \\
\hline \multirow[t]{2}{*}{ TOTAL } & & & & 53 & 16 \\
\hline & & & & & $30 \%$ \\
\hline
\end{tabular}

Mediante el análisis de los índices BMWP/Col y EPT que se realizaron en el río Alpayacu, se pudo determinar que la aptitud del agua se encuentra según Roldan (2003), en una calidad Dudosa cuyo significado es de moderadamente contaminada. La contaminación apreciable se puede evidenciar debido a efectos antropogénicos como la actividad minera y desechos de aguas servidas observados en los sitios de muestreo.

De los resultados observados se puede concluir que los ríos Pindo Mirador y Pindo Grande son los que tienen mayor diversidad y mayor abundancia de los órdenes Plecóptera, Efemeróptera y 
Trichóptera; por lo tanto su calidad de agua es muy buena según el índice BMWP/Col para esta época; sin embargo se puede determinar que los datos no son concluyentes pues estamos hablando de un muestreo en una sola época del año y según Margalef (1983) las comunidades bentónicas varían mucho en las diferentes épocas del año debido a la influencia del clima.

Por otro lado el río Alpayacu en el cual se observa mucha actividad antropogénica la diversidad y la abundancia de los órdenes Plecóptera, Efemeróptera y Tricóptera fue menor, lo cual concluyó según el índice BMWP/Col un estado de calidad ambiental de moderadamente contaminado. Se necesitan más estudios en diversas épocas del año para poder llegar a concluir que el río está contaminado.

Los resultados de ésta investigación permiten determinar la importancia de las micro-cuencas de los ríos Pindo Mirador y Pindo Grande como fuente de agua para las poblaciones que se encuentran río abajo; además de qué modo la presencia o ausencia de organismos bio indicadores (macro invertebrados) muestran la calidad del agua y de los bosques de la micro cuenca.

\section{Agradecimientos}

A los biólogos William Chamorro y Sandra Enríquez por su colaboración en la identificación de los macro invertebrados colectados y a los estudiantes del cuarto semestre de la Carrera de Ingeniería Ambiental, quienes trabajaron en la asignatura de Limnología para que éste documento pueda llevarse a cabo.

\section{Bibliografía}

AOCS Monograph Committee. (1990). Edible Fats and Oils Processing: Basic Principles and Modern Practices. Maastricht: American Oil Chemists Society.

Asociación Mexicana del Amaranto. (2003). Amarantum. Recuperado el 7 de 10 de 2011, de Asociación Mexicana del http://www.amaranto.com.mx/salud/beneficios/beneficios.htm

Astiasarán, I., \& Martínez, A. (2003). Alimentos. Composición y Propiedades. Mexico: McGrawHill.

Bailey, A. (1984). Aceites y grasas industriales. Nueva York: Reverté S.A.

Brennan, J. (2008). Manual del Procesado de los Alimentos. Zaragoza: Acribia S.A.

Carrera, C. y Fierro, K. . (2001). Manual de monitoreo: los macroinvertebrados acuáticos como indicadores de la calidad del agua. Quito: EcoCiencia.

Casp, A., \& Abril, J. (2003). Procesos de Conservación de Alimentos. Madrid: Mundi-Prensa.

Dominguez, E. y Fernández H. (2009). Macroinvertebrados bentónicos sudamericanos. Sistemática y Biología. Tucumán: Fundación Miguel Lillo. 
EPA. (1989). Rapid Bioassessment Protocolos for Use in Stream and Rivers, Benthic Macroinvertebrates and Fish. USA: EPA.

Fernández, H. y Dominguez, E. (2001). Guía para la Determinación de los Artrópodos Bentónicos Sudamericanos. Tucumán: Editorial Universitaria de Tucumán.

Instituto Nacional Autónomo de Investigaciones Agropecuarias (INIAP). (2008). Informe Nacional sobre los Recursos Fitogenéticos para la Agricultura y la Alimentación. Quito: INIAP.

Madrid, A., Cenzano, I., \& Vicente, J. (1997). Manual de Aceites y Grasas Comestibles. Madrid: Mundi - Prensa.

Manzo, V. (2005). Key to the South America genera of Elmidae (Insecta: Coleoptera) with distributional data. Studies of Neotropical Fauna and enviroment, 201-208.

Margalef, R. (1983). Limnología. Barcelona: Editorial Omega.

Merritt, R., Cummins, K. (1988). An Introduction to the Aquatic Insects of North America. USA: Kendall/Huntpublishingcompany.

Moreno, R. (2002). Soporte Nutricional Especial. Bogotá: Panamericana.

NESTLÉ. (2008). Nestlé Sentite Bien. Recuperado el 7 de 3 de 2012, de http://ww1.nestle.com.ar/productos/nestle-maggi-caldos-verduras.html

Roldán, G. (1988). Guía para el estudio de los Macroinvertebrados Acuáticos del Departamento de Antioquia. Bogotá: Editorial Presencia.

Roldán, G. (2003). Bioindicación de la Calidad de Agua en Colombia. Uso del método BMWP/Col. Antioquia: Universidad de Antioquia.

Salles, F. (2006). As Ninfas de Ephemeroptera (Insecta) ocurrentes no Brasil Tese. Vicosa: Universidad Federal de Vicosa.

Ucodep. (24 de 06 de 2011). Quinua, Amaranto, Melloco y Chocho. Un regalo Andino para el mundo. Recuperado el 29 de 09 de 2011, de INIAP: http://www.iniap.gob.ec/

UNIFEM. (1998). Técnicas de Envasado y Empaque. Lima: Asociación Gráfica Educativa.

Velásquez, G. (2006). Fundamentos de Alimentación Saludable. Antioquia: Universidad de Antioquia. 